\title{
Paraneoplastic pityriasis rubra pilaris in association with prostate carcinoma: A case report and literature review
}

\author{
GYULA LÁSZLÓ FEKETE ${ }^{1}$, DANIEL BODA ${ }^{2}$, CONSTANTIN CĂRUNTU ${ }^{3}$ and LÁSZLÓ FEKETE ${ }^{4}$ \\ ${ }^{1}$ University of Medicine and Pharmacy, Dermatology Clinic, 530136 Târgu Mureş; \\ Departments of ${ }^{2}$ Dermatology and ${ }^{3}$ Physiology, 'Carol Davila' University of Medicine and Pharmacy, \\ 050474 Bucharest; ${ }^{4} \mathrm{CMI}$ Dermamed, 530540 Târgu Mureş, Romania
}

Received July 29, 2019; Accepted August 28, 2019

DOI: $10.3892 / \mathrm{etm} .2019 .8169$

\begin{abstract}
Pityriasis rubra pilaris (PRP) is a chronic papulosquamous disorder of unknown etiology, characterized by reddish orange scaly plaques, islands of sparing, palmoplantar keratoderma, and keratotic follicular papules. The disease can be acquired or inherited, being divided into 5 categories: classic adult type, atypical adult type, classic juvenile type, circumscribed juvenile type, and atypical juvenile type. More recently, an HIV-associated type has been added to this classification. The cases of PRP associated with malignancy are unusual. We present a case of a 58-year-old man, with the typical clinical aspect of PRP with a four-month onset of the disease. The histopathological and dermatoscopical findings confirmed the PRP diagnosis. The routine laboratory results were in normal limits, except the number of eosinophils, which was elevated and the number of lymphocytes, which was lower. After a thorough examination within a hematological consultation, the cause of hypereosinophilia remained unknown. An imagistic examination was performed and a prostate hypertrophy was noted. The prostate-specific antigen (PSA) level was found to be increased. The urologic consultation based on clinical, imagistic and microscopic features diagnosed an early stage prostate carcinoma. The conclusion was a paraneoplastic PRP in association with prostate carcinoma. The search in international databases revealed twelve published cases regarding the association of PRP with malignancies. The presented case represents a rare coexistence of PRP with malignancy, particularly with prostate carcinoma, and indicates that PRP can occur as paraneoplastic dermatosis, heralding a
\end{abstract}

Correspondence to: Dr Daniel Boda, Department of Dermatology, 'Carol Davila' University of Medicine and Pharmacy, 8 Eroii Sanitari Avenue, 050474 Bucharest, Romania

E-mail: danielboda.db@gmail.com

Dr Constantin Căruntu, Department of Physiology, 'Carol Davila' University of Medicine and Pharmacy, 8 Eroii Sanitari Avenue, 050474 Bucharest, Romania

E-mail: costin.caruntu@gmail.com

Key words: pityriasis rubra pilaris, paraneoplasia, prostate carcinoma, eosinophilia, prostate-specific antigen malignancy. This case is the first one to present PRP associated with prostate carcinoma. Nonetheless, in the authors' opinion, PRP can be considered a paraneoplastic syndrome; therefore, tumor screening is mandatory in cases presenting this disease.

\section{Introduction}

Pityriasis rubra pilaris (PRP) was first described in 1828 by Tarral and was named by Besnier in 1889. It is a chronic papulosquamous disorder of unknown etiology, characterized by reddish orange scaly plaques, islands of sparing, palmoplantar keratoderma, and keratotic follicular papules (1). The disease can be acquired or inherited (2). Griffiths divided PRP into 5 categories: classic adult type, atypical adult type, classic juvenile type, circumscribed juvenile type, and atypical juvenile type (3). More recently, an HIV-associated type has been added to this classification (4-7). Other reported associations include various infections, autoimmunity, drugs, and malignancies, although their true significance remains unclear (1). Cases of PRP associated with malignancy are unusual. Only a few cases of PRP associated with an underlying malignancy have been documented (8).

\section{Case report}

A 58-year-old man presented an onset of the current disease for four months, with a prior diagnosis of exfoliative dermatitis affecting the extremities, neck, face, scalp, palms and soles. He reported pain, itching, and swelling of the affected areas. The rash appeared to be worst on the hands and feet, and the associated itch made daily activities difficult. His treatment regimen upon admission included systemic and local corticotherapy without significant improvement. The dermatological examination revealed erythematous papillary follicular hyperkeratosis, orange-red and salmon-colored scaly patches and plaques with sharp borders, clearly delimited, sometimes covered by fine, white scales localized on the trunk and neck, confluent in an erythematosquamous plaque on the face and ears. Similar lesions were present on the arms, forearms, knees and thighs. Areas of uninvolved skin, referred to as islands of sparing, were also present (Fig. 1 and 2). The palms and soles had severe confluent orange colored keratoderma. Several painful fissures were present on the soles (Fig. 3). No nail, eye or mucous membrane changes were found. There was moderate pruritus as well. According 


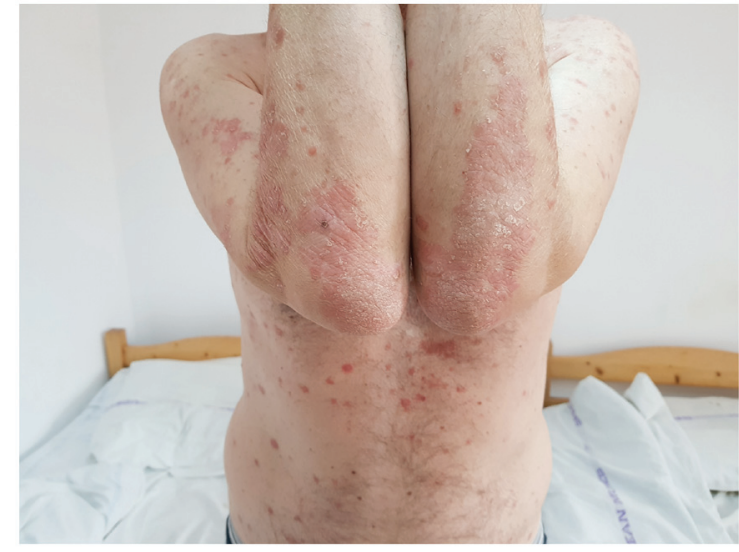

Figure 1. Clinical aspect of trunk anterior area and forearms. Papillary follicular hyperkeratosis, orange-red and salmon-colored scaly patches and plaques covered by fine, white scales.

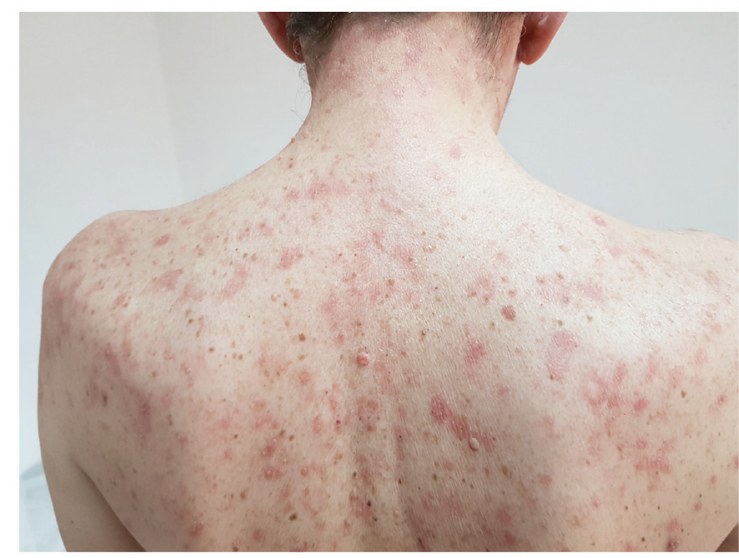

Figure 2. Clinical aspect - dorsal part of trunk. Papillary follicular hyperkeratosis, orange-red and salmon-colored scaly patches and plaques covered by fine, white scales. Islands of sparing.

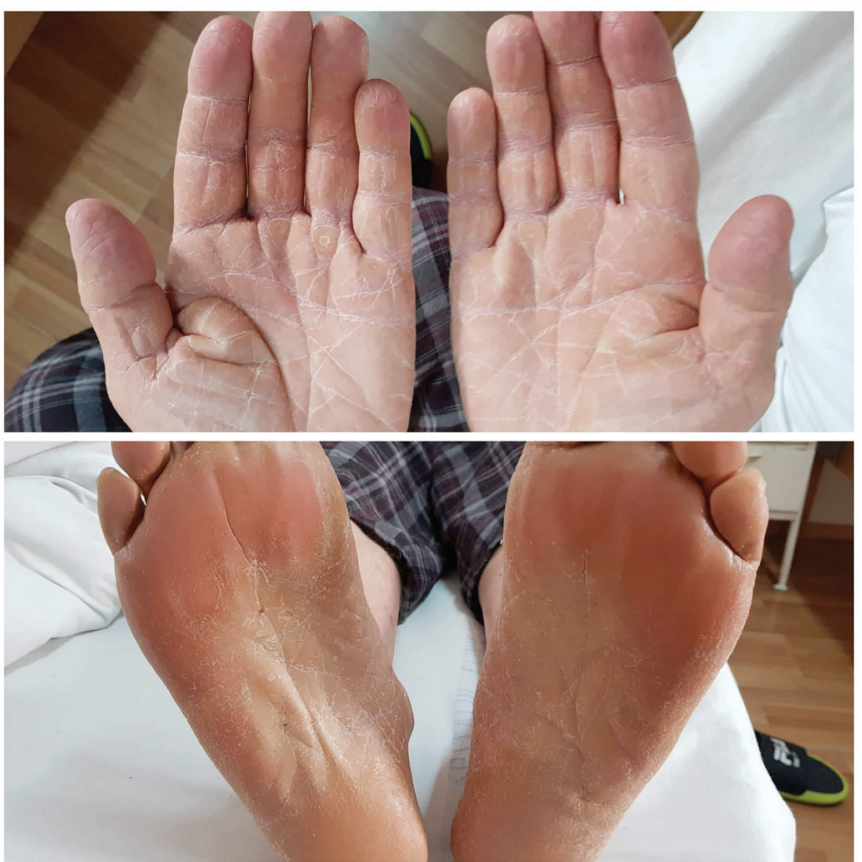

Figure 3. Clinical aspect of the palms and soles. Confluent orange colored keratoderma.

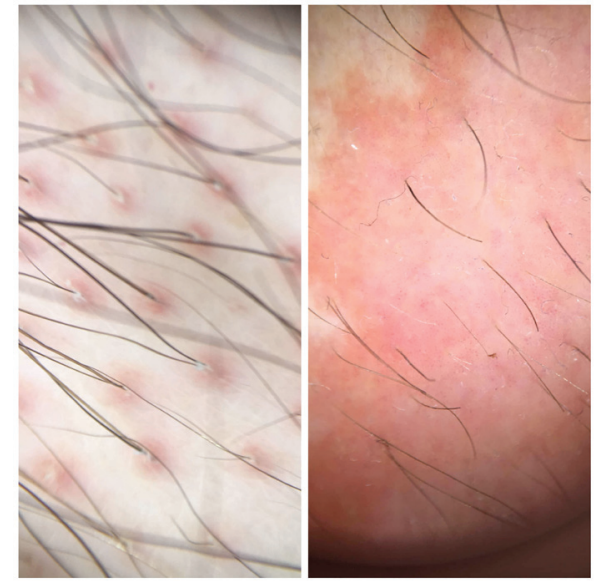

Figure 4. Dermoscopic examination: follicular keratotic plugs and point size vessels with glomerular appearance.

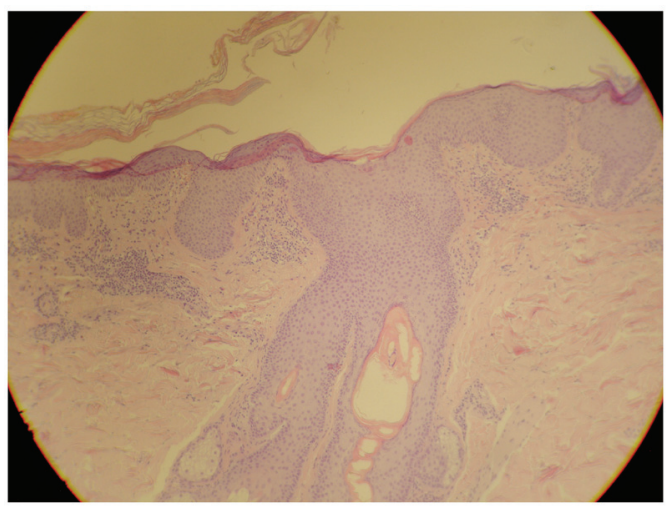

Figure 5. Lamellar hyperkeratosis, focal hypergranulosis, typical follicular plugging with perifollicular parakeratosis, lymphocytic perivascular infiltration, hematoxylin and eosin staining; original magnification, x10.

to the patient, there was no fever, abdominal pain, arthralgia or other relevant subjective symptoms. His medical history was unremarkable. The clinical general examination of systems and organs revealed no relevant findings. The dermoscopic evaluation revealed follicular keratotic plugs and point size vessels with glomerular appearance (Fig. 4). Based on the typical clinical findings, our presumptive clinical diagnosis was PRP. Other diseases, such as psoriasis, keratodermias, acquired ichthyosis or lymphomas were considered as differential diagnosis. A biopsy was performed. The histopathology revealed lamellar hyperkeratosis with alternating orthokeratosis and parakeratosis forming a checkerboard pattern in the stratum corneum, focal hypergranulosis, typical follicular plugging with perifollicular parakeratosis; the presence of a superficial dermal lymphocytic perivascular infiltration confirmed the PRP diagnosis (Fig. 5). Routine laboratory results, including biochemistry and hematology panel, were within normal range, except the number of eosinophils, which was elevated and the number of lymphocytes, which was lower, at a normal range of white blood cell count. The authors found $21.23 \%$ (normal: 0-4\%) eosinophilia and $19.65 \%$ (normal: $25-45 \%$ ) lymphopenia, with an absolute eosinophil count of $1.69 \times 10^{3} / \mu 1$ (normal: $0.05-0.35 \times 10^{3} / \mu 1$ ). After a thorough hematologic examination, the cause of hypereosinophilia remained unclear. The renal function was unimpaired, and the 


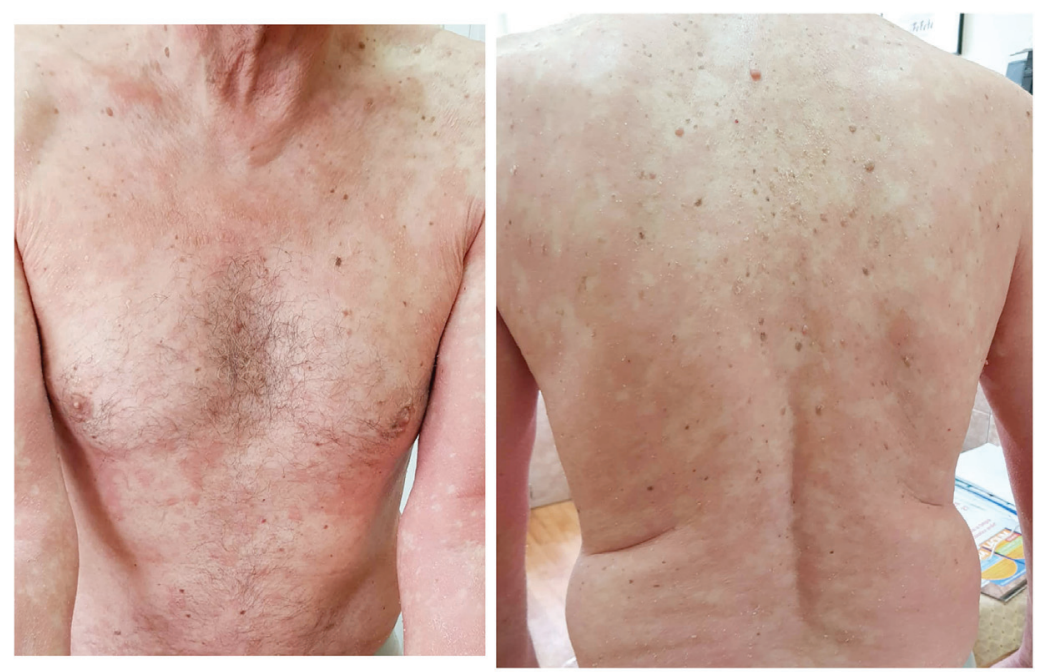

Figure 6. Clinical aspect after one month of treatment.

results of urinalysis were within normal range. No other clinical signs or symptoms and laboratory findings possibly related to an infection or to inflammatory diseases were noted. An imagistic examination was performed in order to exclude underlying diseases. The chest x-ray was negative. An abdominal ultrasound examination revealed only a prostate hypertrophy. The prostate-specific antigen (PSA) level was examined; the result was $4.0 \mathrm{ng} / \mathrm{ml}$. According to the urologic examiner, the PSA level and the ultrasound examination were to be repeated within one month. A treatment with Acitretin $0.5 \mathrm{mg} / \mathrm{kg}$ body weight/day combined with emollients, photoprotection and keratolytics on the palms and soles was started. One month after initiating the treatment, the patient's state presented improvements (Fig. 6). The repeated complete blood cell count with differential showed a decrease of eosinophils to a level of $7.2 \%$. In addition, the PSA level was measured, finding an increased level of $19.2 \mathrm{ng} / \mathrm{ml}$. The urologic consultation based on clinical, imagistic and microscopic features diagnosed an early stage prostate carcinoma. The final diagnosis was a paraneoplastic PRP in association with prostate carcinoma. Written informed consent of the patient was obtained. The Ethics approval was obtained from the Ethics Committee for Research of the University of Medicine and Pharmacy (Târgu Mureş, Romania) (approval nos. 24/2016).

\section{Discussion}

A paraneoplastic syndrome is a syndrome that represents the consequence of a malignancy in the human body. Paraneoplastic syndromes are typical among middle-aged to older patients, when different types of cancers usually occur. Sometimes, the symptoms of paraneoplastic syndromes occur before the diagnosis of a malignancy, but they can be present at the same time as the malignancy or they appear late in the evolution of the cancer. A thorough review of literature was performed using international database search. Available case reports and current review articles were investigated to provide up-to-date information on PRP as paraneoplastic syndrome. According to a search in the PubMed/MEDLINE, Google Scholar and Web of Science databases, twelve published cases on the association of PRP with malignancies were found (2,8-18).
Regarding the documented malignancies associated with PRP the following locations were found: cutaneous in three cases, respiratory tract in three cases, abdominal involved in three cases, two cases with hematological starting and one case with renal involvement. The association of prostate carcinoma with PRP, as in the presented case, has not previously been reported (2,8-18). In cutaneous tumors, there are reported cases of spinocellular, basocellular and Merkel cell carcinomas. Regarding the respiratory tract, in two of the cases it was lung carcinoma and in one case - a laryngeal tumor. In abdominal locations, there were one case of cholangiocarcinoma, one hepatic tumor and one case of liver metastasis with unknown location of the primary tumor. The other remaining cases presented an association of leukemia, Sézary syndrome, and renal carcinoma with PRP. In only two cases (basal cell and spinocellular carcinoma) PRP appeared during the evolution of the cancers. For the rest, PRP was the first clinical sign of a malignancy. The diagnosis of the primary tumors was possible due to the routine clinical, laboratory and imagistic examinations in all of the cases.

The diagnosis of PRP was made based on the clinical and histological findings, as in the present case. The performed dermoscopic examination in this case revealed follicular keratotic plugs and point size vessels with glomerular appearance usually present in PRP (19). No dermoscopic examination was performed by the authors. The increased number of eosinophils found in this case, which decreased under treatment, was not found in the other cases. It is mentioned in literature that eosinophilia can be present in PRP (20). The most likely explanation for the eosinophilia was a reactive process secondary to the extreme inflammatory state. In two cases, PRP had a recalcitrant evolution to retinoid systemic treatment $(8,9)$. In the rest of the cases, the disease reacted to treatment, including in our case. The treatment in all cases was retinoid therapy, except for one case in which locally used steroids were efficient (12). In all cases, the different curative treatments of the malignancies led to healing or marked improvement of the PRP, concluding that PRP can be considered a paraneoplastic syndrome (21-24).

PRP is a rare disease; the incidence may vary from 1 in 5,000 in Britain to 1 in 50,000 in India, affecting both sexes 
equally (3). Most of the cases are acquired, like the present case. The association with malignancies is unusual. This case represented a rare coexistence of PRP with malignancy, particularly with prostate carcinoma, and indicates that PRP can occur as paraneoplastic dermatosis, heralding a malignancy. This is the first case to present PRP associated with prostate carcinoma. The limited number of cases found in literature precludes any meaningful interpretation of data about PRP as paraneoplastic syndrome. Nevertheless, the authors suggest that PRP can be considered a paraneoplastic syndrome; therefore, tumor screening is mandatory in cases presenting this disease.

\section{Acknowledgements}

Professional editing, linguistic and technical assistance performed by Individual Service Provider Irina Radu, certified translator in Medicine and Pharmacy.

\section{Funding}

No funding was received.

\section{Availability of data and materials}

All data generated or analyzed during this study are included in this published article.

\section{Authors' contributions}

GLF was responsible for the clinical management of the patient, the data evaluation and analysis, and the writing of the manuscript. DB contributed to the microscopic examination, the analysis of the data, the corrections and the preparation of the manuscript. CC contributed to the database research and the writing of the manuscript. LF performed the biopsy and contributed to the data analysis, the corrections, the preparation of the manuscript and the database research. All authors read and approved the final manuscript.

\section{Ethics approval and consent to participate}

The Ethics approval was obtained from the Ethics Committee for Research of the University of Medicine and Pharmacy (Târgu Mureş, Romania) (approval nos. 24/2016). Written informed consent of the patient was obtained.

\section{Consent for publication}

Written informed consent of the patient has been obtained.

\section{Competing interests}

The authors declare that they have no competing interests.

\section{Authors' information}

GLF: Associate Professor of Dermatology, Dermatology Department, University of Medicine and Pharmacy, Dermatology Clinic, Târgu Mureş, Romania.

\section{References}

1. Wang D, Chong VC, Chong WS and Oon HH: A review on pityriasis rubra pilaris. Am J Clin Dermatol 19: 377-390, 2018.

2. Batinac T, Kujundzić M, Peternel S, Cabrijan L, Troselj-Vukić B and Petranović D: Pityriasis rubra pilaris in association with laryngeal carcinoma. Clin Exp Dermatol 34: e917-e919, 2009.

3. Griffiths WA: Pityriasis rubra pilaris. Clin Exp Dermatol 5: $105-112,1980$

4. Auffret N, Quint L, Domart P, Dubertret L, Lecam JY and Binet O: Pityriasis rubra pilaris in a patient with human immunodeficiency virus infection. J Am Acad Dermatol 27: 260-261, 1992.

5. Blauvelt A, Nahass GT, Pardo RJ and Kerdel FA: Pityriasis rubra pilaris and HIV infection. J Am Acad Dermatol 24: 703-705, 1991.

6. Martin AG, Weaver CC, Cockerell CJ and Berger TG: Pityriasis rubra pilaris in the setting of HIV infection: Clinical behaviour and association with explosive cystic acne. Br J Dermatol 126: 617-620, 1992

7. Miralles ES, Núñez M, De Las Heras ME, Pérez B, Moreno R and Ledo A: Pityriasis rubra pilaris and human immunodeficiency virus infection. Br J Dermatol 133: 990-993, 1995.

8. Bar-Ilan E, Gat A, Sprecher E and Zeeli T: Paraneoplastic pityriasis rubra pilaris: Case report and literature review. Clin Exp Dermatol 42: 54-57, 2017.

9. Remedios IM, Jensen JD, Beckum K, McKay K and Kissel R: Paraneoplastic pityriasis rubra pilaris as the presenting manifestation of metastatic squamous cell carcinoma. J Drugs Dermatol 13: 610-612, 2014.

10. Kurzydlo AM and Gillespie R: Paraneoplastic pityriasis rubra pilaris in association with bronchogenic carcinoma. Australas $\mathbf{J}$ Dermatol 45: 130-132, 2004.

11. Garretson CB, Machan ML, Krejci-Manwaring J, Aires D and Tonkovic-Capin V: Letter: Adenocarcinoma of the lung associated with pityriasis rubra pilaris. Dermatol Online J 17: 14-17, 2011.

12. Sánchez-Regaña M, López-Gil F, Salleras M and Umbert P: Pityriasis rubra pilaris as the initial manifestation of internal neoplasia. Clin Exp Dermatol 20: 436-438, 1995.

13. Batchelor RJ, Yung A, Merchant W and Goodfield MJ: Pityriasis rubra pilaris as the initial presentation of renal cell carcinoma? Clin Exp Dermatol 30: 442-443, 2005.

14. Sharma S, Weiss GR and Paulger B: Pityriasis rubra pilaris as an initial presentation of hepatocellular carcinoma. Dermatology 194: 166-167, 1997.

15. Reinhardt LA and Rosen T: Pityriasis rubra pilaris as the initial manifestation of leukemia. Cutis 31: 100-102, 1983.

16. Roger J, Burg G, Miller K and Lanz U: Pityriasis rubra pilarisartiges Vorstadium eines Sezary-Syndroms. Syndroms (Pityriasis rubra pilaris the precursor of a Sezaryís syndrome). Z Hautkr 66: 1046-1050, 1991. (In German).

17. Tannenbaum CB, Billick RC and Srolovitz H: Multiple cutaneous malignancies in a patient with pityriasis rubra pilaris and focal acantholytic dyskeratosis. J Am Acad Dermatol 35: 781-782, 1996.

18. Huynh NT, Hunt MJ, Cachia AR and Veness MJ: Merkel cell carcinoma and multiple cutaneous squamous cell carcinomas in a patient with pityriasis rubra pilaris. Australas J Dermatol 43: 48-51, 2002.

19. Abdel-Azim NE, Ismail SA and Fathy E: Differentiation of pityriasis rubra pilaris from plaque psoriasis by dermoscopy. Arch Dermatol Res 309: 311-314, 2017.

20. Price L and Lesesky E: Pityriasis rubra pilaris and severe hypereosinophilia. Cutis 100: E6-E7, 2017.

21. Fekete GL, Cotoi OS and Fekete JE: Multiple nodular cutaneous metastases as the first clinical sign of signet ring cell gastric carcinoma: Case report. Acta Dermatovenerol Croat 20: 34-37, 2012.

22. Neagu M, Caruntu C, Constantin C, Boda D, Zurac S, Spandidos DA and Tsatsakis AM: Chemically induced skin carcinogenesis: Updates in experimental models (Review). Oncol Rep 35: 2516-2528, 2016.

23. Boda D: Cellomics as integrative omics for cancer. Curr Proteomics 10: 237-245, 2013.

24. Neagu M, Constantin C, Tanase C and Boda D: Patented biomarker panels in early detection of cancer. Recent Pat Biomark 1: 10-24, 2011.

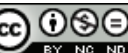

This work is licensed under a Creative Commons Attribution-NonCommercial-NoDerivatives 4.0 International (CC BY-NC-ND 4.0) License. 